\title{
Research of a Weighted Multi-link Load Balance Network Model Based on P2P
}

\author{
Jiao Li ${ }^{1,2}$, Gongqian Liang ${ }^{1}$, Tianshi Liu ${ }^{2}$ \\ ${ }^{1 .}$ School of Management, Northwestern Polytechnical University, Xi'an 710072, China; \\ 2. School of Computer Science, Xi'an Shiyou University, Xi'an 710065, China
}

\begin{abstract}
To solve P2P network load balance problem, this paper researches a weighted multi-link load balance network model based on P2P. Based on the model, it analyzes P2P communication algorithms, and then gives communication performance evaluation parameters. Finally, it summarizes the characteristics of the model. This model considers two important factors, namely, the node communication link number and the communication weight. In addition, the communication process is concurrent and without intermission, so the efficiency of network model is high. This model provides the theory reference for $P 2 P$ network route decision scheme.
\end{abstract}

Keywords-communication weight; multi-link; communication algorithm; load balance; evaluation parameters.

\section{INTRODUCTION}

With network applications becoming increasingly diverse, users prefer multimedia information, such as audio, graphic, video, to text-based information, and require high quality multimedia information. This results in a large amount of network traffic, so network congestion problems have become increasingly prominent. The traditional FTP mode depends heavily on centre node, so in large-scale communication system or the larger amount of data transmission, the centre node may be failure or malfunction and the network transmission bottleneck may arise. P2P load balance method has become one of the hottest research points. Present researches of P2P load balance method are specific to a particular application context, and don't theoretically and systematically analyze P2P load balance problem, so there are limitations[1-3]. We have studied some algorithms, such as Hierarchical 2PC protocol algorithm, Kruskal communication tree algorithm, BFI algorithm, Multi-link communication tree algorithm, WFI algorithm, JFI algorithm, FIN algorithm, but the communication factors are not considered comprehensively, and these algorithms don't systematically analyze and summarize P2P load balance network model[4-10]. This paper studies a weighted multi-link load balance network model based on P2P.

\section{MODEL AND ALGORITHM}

\section{A. The weighted multi-link load balance network model}

In P2P networks, peer stands for node, which is a single computer or server group. The communication speed and the communication cost between nodes differ widely, that is to say, the communication weight is not equal. The node's different hardware configurations such as CPU, memory and other hardware, and network bandwidth result in that node's communication capabilities differ, that is to say, the node communication link number is not equal. Due to these communication factors in $\mathrm{P} 2 \mathrm{P}$ network, besides the distribution and dynamic of P2P network, there is certain difficulty in achieving P2P network load balance, so how to construct a model P2P based on these communication factors is the first thing we should do, and then we should study P2P communication algorithms based on the model. The model studied in this paper considers both the communication weight and the node communication link number. Therefore, the weighted multi-link load balance network model based on P2P is classified into four categories: a single-link equal weight communication model, a single-link non-equal weight communication model, a multi-link equal weight communication model and a multi-link non-equal weight communication model.

In design of the model, the node itself factor and the mutual factor between nodes, namely the node communication link number and the communication weight, are considered. In this model, nodes can develop their maximum communication capacity to concurrently undertake communication task, and the communication route between nodes, which's weight is as small as possible, is choosed. Therefore, P2P network can achieve load balance and the efficiency of communication model is high[4].

\section{B. Algorithm analysis based on model}

Different communication algorithms are proposed based on communication models. Table I shows the communication models and algorithms.

TABLE I. THE COMMUNICATION MODELS AND ALGORITHMS

\begin{tabular}{|c|c|c|}
\hline No. & communication models & algorithms \\
\hline 1 & single-link equal weight & $\begin{array}{l}\text { Hierarchical 2PC protocol } \\
\text { algorithm }\end{array}$ \\
\hline 2 & $\begin{array}{l}\text { single-link non-equal } \\
\text { weight }\end{array}$ & $\begin{array}{l}\text { Kruskal communication tree } \\
\text { algorithm } \\
\text { BFI algorithm }\end{array}$ \\
\hline 3 & multi-link equal weight & $\begin{array}{l}\text { Multi-link communication tree } \\
\text { algorithm }\end{array}$ \\
\hline 4 & $\begin{array}{l}\text { multi-link } \\
\text { weight }\end{array}$ & $\begin{array}{l}\text { WFI algorithm } \\
\text { JFI algorithm } \\
\text { FIN algorithm }\end{array}$ \\
\hline
\end{tabular}


Firstly, we study algorithm from the simplest case, so without considering any communication factors we propose the hierarchical 2PC protocol algorithm that belongs to the single-link equal weight communication model. It can get optimal P2P communication tree [5].

Secondly, the Kruskal communication tree algorithm [6] and the branch first communication algorithm (BFI) are proposed, which only consider weight impact factor in constructing communication tree, belong to the single-link non-equal weight communication model. Kruskal communication tree algorithm first and foremost selects a smaller edge weight added to communication tree, and BFI algorithm preferentially selects the edge weight that makes the branch communication time shorter. The simulation results show that BFI algorithm is superior to Kruskal communication tree algorithm in communicate efficiency in most cases [7].

Then, we study the multi-link communication tree algorithm that belongs to the multi-link equal weight communication model, which only considers the node communication link number. In constructing communication tree, this algorithm uses the greedy thinking to preferentially select the strong communication nodes as the communication sources. Simulation results indicate that a multi-link communication tree is superior to a single-link communication tree in concurrent communication time, communication tree node utilization rate and communication concurrency [8].

Finally, we study the most complex situation, we propose three algorithms, the weight first communication tree algorithm (WFI), the joint first communication tree algorithm (JFI) and the factors integrated communication tree algorithm (FIN) which belong to the multi-link nonequal weight communication model. WFI algorithm preferentially chooses node which has the smallest communication weight to add to the communication tree, and JFI algorithm preferentially chooses node which has the largest communication link number to add to the communication tree. FIN algorithm integrates the node communication link number and the communication weight, and preferentially selects node which has the smallest integration factor. This algorithm chooses the minimum of branch communication time as objective function in order to shorten communication time and to reduce communication latency. A large number simulation results indicate that FIN algorithm is superior to WFI algorithm and JFI algorithm in communicate efficiency [9-10].

In the research process of $\mathrm{P} 2 \mathrm{P}$ communication tree algorithms, we constantly join the communication impact factors, and gradually improve the communication models. Finally, we get the multi-link non-equal weight communication model. However, the solutions given by JFI algorithm, WFI algorithm, and FIN algorithm are not optimal, and the communication tree construction process is static, can not search for better solution dynamically and heuristically. Therefore, JFI algorithm, WFI algorithm, and FIN algorithm can be applied to the ant colony optimization. Using the positive feedback of ant colony algorithm, we search dynamically for the better solution or even the optimal solution of $\mathrm{P} 2 \mathrm{P}$ communication tree in order to further optimize the P2P communication tree. These studies are being processed. In early study we apply BFI algorithm to ant colony optimization, and communicate efficiency is improved by $10 \%$ [11].

\section{COMMUNICATION PERFORMANCE EVALUATION PARAMETERS}

We can construct the P2P communication tree algorithm based on the weighted multi-link load balance network model, and the algorithm gives a communication route decision scheme. Evaluating the strengths and weaknesses of scheme's performance has multiple parameters, such as concurrent communication time, communication concurrency, communication tree node utilization rate, average end to end delay, etc.

\section{A. Concurrent communication time}

In P2P communication tree, according to concurrent communication rules, the time, spent in communication from the source node $v_{1}$ to the last node received the data, is called concurrent communication time. Indicated by $f(t)$. In practice, people are more concerned about the communication time, so the concurrent communication time is the most important parameter in communication performance evaluation.

\section{B. Communication concurrency}

In order to clearly describe the degree of concurrent communication, the concept of communication concurrency is introduced. It is the maximum number of communication tasks executed at the same time. Indicated by $C_{d}$.

In the multi-link equal weight communication model, the communication concurrency is

$$
C_{d}=\operatorname{Max}\{\operatorname{Task}(f(t)-1), \operatorname{Task}(f(t))\}
$$

Where $\operatorname{Task}(t)$ is the number of tasks executing in the time $t$.

\section{Communication tree node utilization rate}

For the communication tree of $N$ nodes, the ratio of source nodes to $N$ is the communication tree node utilization rate, noted as $U r$, Then

$$
U r=\frac{\operatorname{Num}\left(\left\{v_{i} \mid \operatorname{task}\left(v_{i}\right)>0\right\}\right)}{N}
$$

Where $\operatorname{Task}\left(v_{i}\right)$ is the number of node $v_{i}$ as traffic source sending data, $\operatorname{Num}(X)$ indicates the element number of set $X$.

\section{Average end to end delay}

For the communication tree of $N$ nodes, the ratio of the sum of end to end delay from source node to each node to $N$ is called Average End-to-end Delay, Indicated by AED. Then 


$$
A E D=\frac{f_{A}(t)}{N}
$$

Where $f_{A}(t)$ is the accumulative communication time, AED is the average time of each node received data in P2P communication tree.

\section{CHARACTERISTICS OF MODEL}

The weighted multi-link load balance network model abstracts the P2P network as tree structure logically, and doesn't take into account the heterogeneity and the actual distribution of the physical network. Algorithms based on this model can give the communication route decision scheme. The characteristics of this model are as follows:

i) High concurrency, In the communication model, multiple communication tasks are executed simultaneously. This model can maximize the capability of communication nodes, and the entire communication process is without intermittent concurrent communication. For the communication tree based on the single-link equal weight communication model, if the number of nodes is $N$, its concurrent communication time is $\mathrm{O}(\operatorname{lnN})$, and its accumulative communication time is $\mathrm{O}(\mathrm{N})$. It shows that the higher concurrency, the more communication tasks finished in the unit of time and the higher the efficiency [5].

ii) Strong robustness, As a result of hierarchical structure, the communication does not depend on a single communication node. When some nodes are under attack, even failure, they have little effect on the entire communication process. Therefore, this model has strong robustness.

iii) Effective load balance, In P2P communication tree, nodes have different communication capability and communication weights between nodes are different. JFI algorithm based on the multi-link non-equal weight communication model, rationally assigns communication tasks to nodes according to the node communication link number and the communication weight. That the larger communication link number of the node, the more tasks it will afford, reflects that able people should do more work. Therefore, this model effectively improves communication efficiency, and achieves load balance of P2P network.

\section{CONCLUSION}

This paper researched a weighted multi-link load balance network model based on the communication weight and the node communication link number. This model can effectively achieve load balance. But in algorithm simulation, the node communication link number and the communication weight are given as constant, however, the node communication link number and the communication weight change with the bandwidth and network load, so they are variables. In the follow-up research, we consider impact factors as variables to improve communication model. In addition, we can not prove that the solutions given by algorithms studied above are optimal solutions, so we should apply artificial intelligence algorithm to solve this problem in future research.

\section{ACKNOWLEDGEMENTS}

The research work was supported by Natural Science Foundation of Shaanxi Provincial under Grant No. 2010JM8032, 2012JQ8040, 2012JM8037.

\section{REFERENCES}

[1] Xiong Wei, Xie Dong-Qing, Jiao Bing-Wang, Liu Jie, Self-Adaptive Load Balancing Method in Structured P2P Protocol, Journal of Software, 20(3), pp.660-670, 2009.

[2] Zhu Bin, Zeng Xiao-ping, Chen Li, Liao Hong-yun, Yan Fang, Research on Adaptive Load Balancing Routing Algorithm, Journal of Xidian University, 38(5), pp.120-127, 2011.

[3] Lu Chui-wei, Li Zhi-tang, Lin Huai-qin, Huang Qing-feng, Zhang Ye-jiang, Dynamic Load-balancing Algorithm on Structured P2P Network, Computer Science, 36(11), pp.43-46, 2009.

[4] Li Jiao, Liu Tian-shi. Research of a Multi-link Concurrent P2P Communication Model. Applied Mechanics and Materials. Vols 263266, pp:1012-1025, 2013.

[5] Liu Tian-shi, Zhao Song-zheng, Study on a Communication Algorithm of Hierarchical 2PC Protocol, Computer Engineering, 30(6), pp.104-105\&141, 2004.

[6] Liu Tian-shi, Zhao Song-zheng, P2P Distributed Database System, Science Press. Beijing: 2007.

[7] Liu Tian-shi, Li Jiao, Cao Qing-nian. Study on a Network Communication Optimization Algorithm of P2P Mode. Proceedings of WISM'09-AICI'09 IEEE Computer Society Order Number P3816, volume II, pp. 212-217,2009.

[8] Li Jiao, Liu Tian-shi,. Study on a P2P Communication Tree Optimization Algorithm Based on Multi-link, Journal of Northwest University(Natural Science Edition), 40(6), pp.970-974, 2010.

[9] Liu Tian-shi, Yang Kun-yi, Li Jiao. Study on a Concurrent Communication Tree Algorithm of P2P Multi-link Mode, Proceedings of ICMT, volume VI, pp.2034-2038, 2010.

[10] Liu Tian-shi, Zhang Liu-mei, Cheng Guo-jian, Li Jiao, YANG Kunyi, FIN multicast optimization algorithm in P2P communication, Application Research of Computers, 30(5),pp.1464-1466\&1491, 2013.

[11] Liu Tian-shi, Li Jiao, Study on P2P Communication Tree Optimization Method Based on Ant Colony Algorithm, Advances in China Artificial Intelligence, 10, pp.458-461, 2009. 